\title{
Unusual fatty acid composition of storage lipids in the bresilioid shrimp Rimicaris exoculata couples the photic zone with MAR hydrothermal vent sites
}

\author{
David W. Pond ${ }^{1, *}$, Andrey Gebruk ${ }^{2,3}$, Eve C. Southward ${ }^{2}$, Alan J. Southward ${ }^{2}$, \\ Anthony E. Fallick ${ }^{4}$, Michael V. Bell ${ }^{5}$, John R. Sargent ${ }^{5}$ \\ ${ }^{1}$ Southampton Oceanography Centre, Empress Dock, Southampton SO14 3ZH, United Kingdom \\ ${ }^{2}$ Marine Biological Association, Citadel Hill, The Hoe, Plymouth PL1 2PB, United Kingdom \\ ${ }^{3}$ Shirshov Institute of Oceanology, Russian Academy of Sciences, Nakhimovsky pr. 36, Moscow 117851, Russia \\ ${ }^{4}$ Scottish Universities Research and Reactor Centre, East Kilbride, Glasgow G75 OQF, United Kingdom \\ ${ }^{5}$ Institute of Aquaculture, University of Stirling, Stirling FK9 4LA, United Kingdom
}

\begin{abstract}
Lipid and stable carbon isotope analyses of Rimicaris exoculata, the dominant bresilioid shrimp found at the MAR (Mid-Atlantic Ridge) vent sites, have indicated that these animals possess a highly unusual storage lipid composition. The dominant neutral lipid classes, triacylglycerols and wax esters, contained very high levels of polyunsaturated fatty acids (PUFA, up to $89 \%$ of neutral lipid fatty acids). Gas chromatography isotope ratio mass spectrometry (GC-IRMS) analysis of the PUFA from neutral lipid gave $\delta^{13} \mathrm{C}$ (v-PDB) values of -17.6 to $-27.1 \%$, which is within the range expected for a photosynthetic origin for these compounds. Fatty acid analyses of bacterial/detrital material collected from the vent sites contained only very low amounts of PUFA. It is clear from these findings that $R$, exoculata has evolved a highly specialized lipid metabolism which allows it to store substantial amounts of PUFA during its early planktotrophic life stages. These PUFA reserves will be subsequently mobilized to enable growth and maturation of the shrimp on return to a suitable vent site and are therefore an important factor allowing $R$. exoculata to inhabit deep sea vent ecosystems.
\end{abstract}

KEY WORDS: Hydrothermal vent shrimp · Nutrition · Lipid metabolism · Wax ester · PUFA

\section{INTRODUCTION}

The food sources of Rimicaris exoculata, the dominant shrimp inhabiting the Mid-Atlantic Ridge (MAR) hydrothermal vent sites, have been studied for a number of years. These studies have suggested that the shrimp derives its nutritional requirements from strains of chemoautotrophic bacteria that either encrust the walls of the vent chimneys, or encrust the carapace of the shrimp itself (Van Dover 1995, Gebruk et al. 1997 and references therein). More recently it has been found that bacterial populations within the gut of $R$. exoculata also exhibit high rates of chemoautotrophic production and could constitute an additional food source (Polz et al. 1998). However, for higher marine

•E-mail: dwpo@soc.soton.ac.uk animals, there are important nutritional limitations for a diet that almost exclusively comprises bacteria. Some marine organisms including decapod shrimp require certain essential polyunsaturated fatty acids (PUFA) to be supplied in their diet (Kanazawa et al. 1979). These compounds have crucial roles in the structure and functioning of cell membranes, and the animals require substantial amounts during periods of growth and reproduction (Jónasdóttir 1994, Sargent et al. 1995, Pond et al. 1996). In the marine ecosystem as a whole, the bulk of PUFAs is produced by phytoplankton (Sargent \& Henderson 1995). Bacteria are generally incapable of synthesizing polyunsaturated fatty acids as they tend to lack the necessary oxygen-dependent desaturases. In recent years some strains of PUFA-producing heterotrophic bacteria have been isolated from a range of marine environments (Russell \& 
Nichols 1999), but, as yet, none have been identified at MAR vent sites. It is therefore unlikely that the chemoautotrophic bacteria on which $R$. exoculata feeds at the vent sites contain sufficient amounts of PUFA for the nutrition of this shrimp. The key question we examined here is how the populations of vent shrimp inhabiting the MAR vent sites have solved this apparent nutritional paradox.

Previous studies have shown that the early life stages of MAR vent shrimps can accumulate PUFAs derived from phytoplankton (Pond et al. 1997a,b,c). During :these early stages the larval shrimp are components of the deepwater plankton community and their lipid composition is typical of bathypelagic organisms, which are exclusively reliant on organic carbon originating from the surface layers of the ocean (Pond et al. 1997a, in press). Here we extend the previous work of Pond et al. (1997a,b,c, Dixon et al. 1998) by analyzing the lipid and stable isotope composition of different developmental stages of Rimicaris exoculata from a variety of MAR vent sites.

\section{METHODS}

Sampling. Specimens of Rimicaris exoculata were collected by slurp-gun from 6 sites along the MAR during a cruise of RV 'Atlantis' and the submersible 'Alvin', when all known sites of hydrothermal activity were visited. Table 1 summarizes the position and depth of the sites where $R$. exoculata occurred. After arrival of shrimp on board ship, animals were quickly sized (total length) and identified in terms of 3 maturity stages. In the first category small 'orange' shrimp were between 16 and $18 \mathrm{~mm}$ in length, bright orange in color and contained substantial lipid reserves which were easily visible under the carapace. These shrimp were initially referred to as Iorania concordia, originally described as a separate species by Vereshchaka (1996). However, it is now established that these small orange shrimp are a juvenile form of $R$. exoculata (Shank et al. 1998). We analyzed 2 further categories of $R$. exoculata, i.e. small adults (25 to $26 \mathrm{~mm}$ ), which

Table 1. Rimicaris exoculata. Locations of the Mid-Atlantic Ridge (MAR) vent sites

\begin{tabular}{|lccc|}
\hline Site & Latitude N & Longitude W & Depth (m) \\
\hline Lucky Strike & $37^{\circ} 17.5^{\prime}$ & $32^{\circ} 16.5^{\prime}$ & $1600-1720$ \\
Rainbow & $36^{\circ} 14.0^{\prime}$ & $33^{\circ} 54.0^{\prime}$ & $2305-2350$ \\
Broken Spur & $29^{\circ} 10.0^{\prime}$ & $43^{\circ} 10.4^{\prime}$ & $3050-3060$ \\
TAG & $26^{\circ} 08.0^{\prime}$ & $44^{\circ} 49.5^{\prime}$ & $3640-3660$ \\
Snakepit & $23^{\circ} 22.2^{\prime}$ & $44^{\circ} 57.0^{\prime}$ & $3510-3560$ \\
Logatchev & $14^{\circ} 44.9^{\prime}$ & $44^{\circ} 58.3^{\prime}$ & $3010-3038$ \\
\hline
\end{tabular}

were a whitish gray in color, and larger adults (29 to $57 \mathrm{~mm}$ ). Neither the small nor larger adults contained the substantial pigmented lipid reserves found in the juveniles. Immediately after characterization, shrimp were dissected and the tissue samples transferred to chloroform:methanol $(2: 1 \mathrm{v} / \mathrm{v})$ and stored at $-20^{\circ} \mathrm{C}$ until analysis in the UK.

Lipid analyses. Samples of tissue contained in the chloroform:methanol were initially homogenized before being filtered through a prewashed (chloroform:methanol 2:1, v/v) Whatman No. 1 paper filter. Total lipid was extracted following Folch et al. (1957) and dried under nitrogen. Aliquots of total lipid $(15 \mu \mathrm{g})$ were separated into individual lipid classes of neutral lipid by high performance thin layer chromatography (HPTLC) using a hexane:diethyl ether:acetic acid (90:10:1 v/v/v) soivent system. Lipid classes were visualized by spraying the plates with $8 \%(\mathrm{v} / \mathrm{v})$ phosphoric acid containing $3 \%(\mathrm{w} / \mathrm{v})$ copper acetate followed by charring at $160^{\circ} \mathrm{C}$ for $15 \mathrm{~min}$ and then quantified by scanning densitometry (Olsen \& Henderson 1989).

Remaining samples of total lipid were separated into polar lipid, triacylglycerol and wax ester by thin-layer chromatography (TLC), as above, and visualized under UV light after spraying with 2,7-dichlorofluorescein in $97 \%(\mathrm{v} / \mathrm{v})$ methanol. Individual lipid classes were then transesterified in methanol containing $1.5 \%(\mathrm{v} / \mathrm{v})$ sulphuric acid for $16 \mathrm{~h}$ at $50^{\circ} \mathrm{C}$, and the resulting fatty acid methyl esters were purified by TLC in a hexane:diethyl ether:acetic acid solvent system $(90: 10: 1 \mathrm{v} / \mathrm{v} / \mathrm{v})$. For the wax ester fraction, free fatty alcohols were recovered separately from the TLC plates and converted to their acetate derivatives following Farquhar (1962). Purified fatty acid methyl esters and fatty alcohol acetate derivatives were analyzed separately by gas chromatography (GC) on a Carlo Erba (6000 vega series) fitted with a BP20 fused silica capillary column $(50 \mathrm{~m}$ by $0.32 \mathrm{~mm}$ internal diameter). Hydrogen was used as the carrier gas. Fatty acids were identified by reference to standards of known composition and by GC-MS as described in Pond et al. (1997b, in press).

GC-IRMS analyses. Stable carbon isotope ratios $\left({ }^{13} \mathrm{C} /{ }^{12} \mathrm{C}\right)$ were determined for individual fatty acids and fatty alcohols by GC-combustion IRMS (isotope ratio mass spectrometry) with a VG Isochrom II instrument equipped with a column similar to that described in the preceding section (Eakin et al. 1992). The $\delta^{13} \mathrm{C}$ value of the methanol derivatization reagent was determined by conventional closed-tube combustion (Sofer 1980), and the contribution of derivatized carbon to specific fatty acids was calculated by rearranging the equation from Abrajano et al. (1994):

$$
\delta^{13} \mathrm{C}_{\mathrm{FA}}=\frac{\delta^{13} \mathrm{C}_{\mathrm{FAME}}-(1-x) \delta^{13} \mathrm{C}_{\mathrm{CH}_{3} \mathrm{OH}}}{x}
$$


where: $\delta^{13} \mathrm{C}_{\mathrm{FA}}$ is the isotopic composition of the free fatty acid, $\delta^{13} \mathrm{C}_{\mathrm{FAME}}$ is the isotopic composition of the fatty acid methyl ester, $\boldsymbol{x}$ is the fractional carbon contribution of the free fatty acid to the ester and $\delta^{13} \mathrm{C}_{\mathrm{CH}_{3} \mathrm{OH}}$ is the isotopic composition of the methanol derivatization reagent. All isotope data are reported as $\delta^{13} \mathrm{C}(\% \mathrm{v}-$ PDB) and precision is $\pm 0.4 \%$ or better.

\section{RESULTS}

\section{Lipid class composition}

The lipid class composition of the 3 categories of Rimicaris exoculata are summarized in Fig. 1. The lipids of the juvenile shrimp comprised predominantly wax esters ( $56 \%$ of total lipid) with only comparatively minor amounts of triacylglycerol $(10 \%$, Fig. 1$)$. Small adult $R$. exoculata contained both wax ester and triacylglycerols in -similar proportions ( 31 and $24 \%$ ), whilst the lipid composition of adult $R$. exoculata was dominated by triacylglycerols and polar lipid (Fig. 1).

\section{Fatty acid and fatty alcohol composition}

Bacterial/detrital material collected from vent sites contained only very low proportions of PUFA (approx.

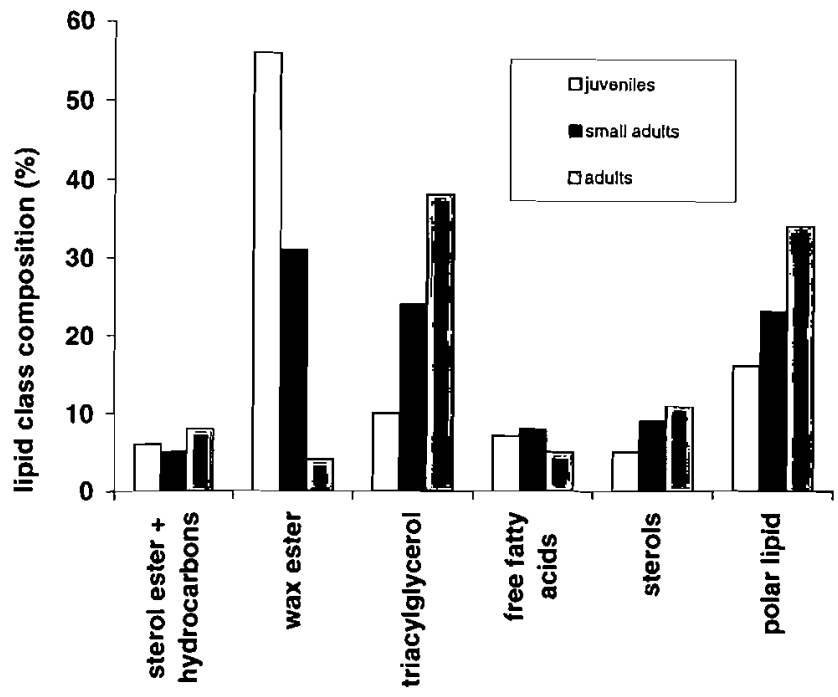

Fig. 1. Rimicaris exoculata. Lipid class composition (\% wt). Values are means of all shrimp in each of the 'orange' juvenile ( $n=4)$, small adult $(n=2)$ and adult $(n=6)$ categories

$1 \%$ ), comprising mostly saturated and monounsaturated fatty acids (Table 2). By contrast the fatty acid compositions of the juvenile Rimicaris exoculata were dominated by the polyunsaturated fatty acid 22:6(n-3), with wax ester consistently more enriched in this fatty acid than polar lipid (Table 3). Other major fatty

Table 2. Fatty acid composition (\%) of total lipid extracted from filamentous free-living bacteria (fb) and detrital material (dm) collected from MAR vent sites ("collected from mussel shells; $a, b$, c refer to replicates)

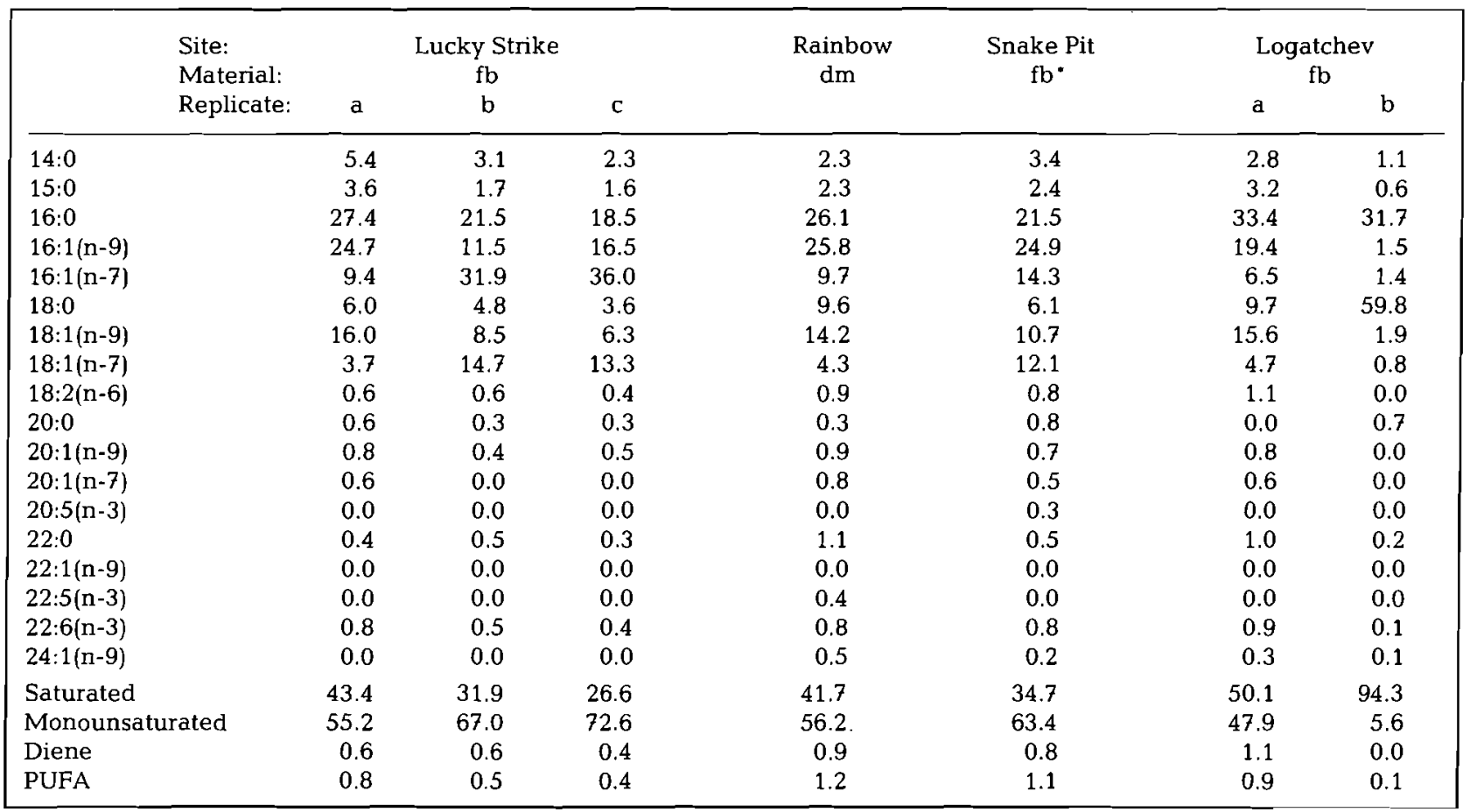


Table 3. Rimicaris exoculata. Fatty acid and fatty alcohol composition (\%) of the major lipid classes in 'orange' (16 to $18 \mathrm{~mm}$ ) and small adult ( 26 to $28 \mathrm{~mm}$ ) $R$. exoculata containing predominantly wax ester neutral lipid reserves

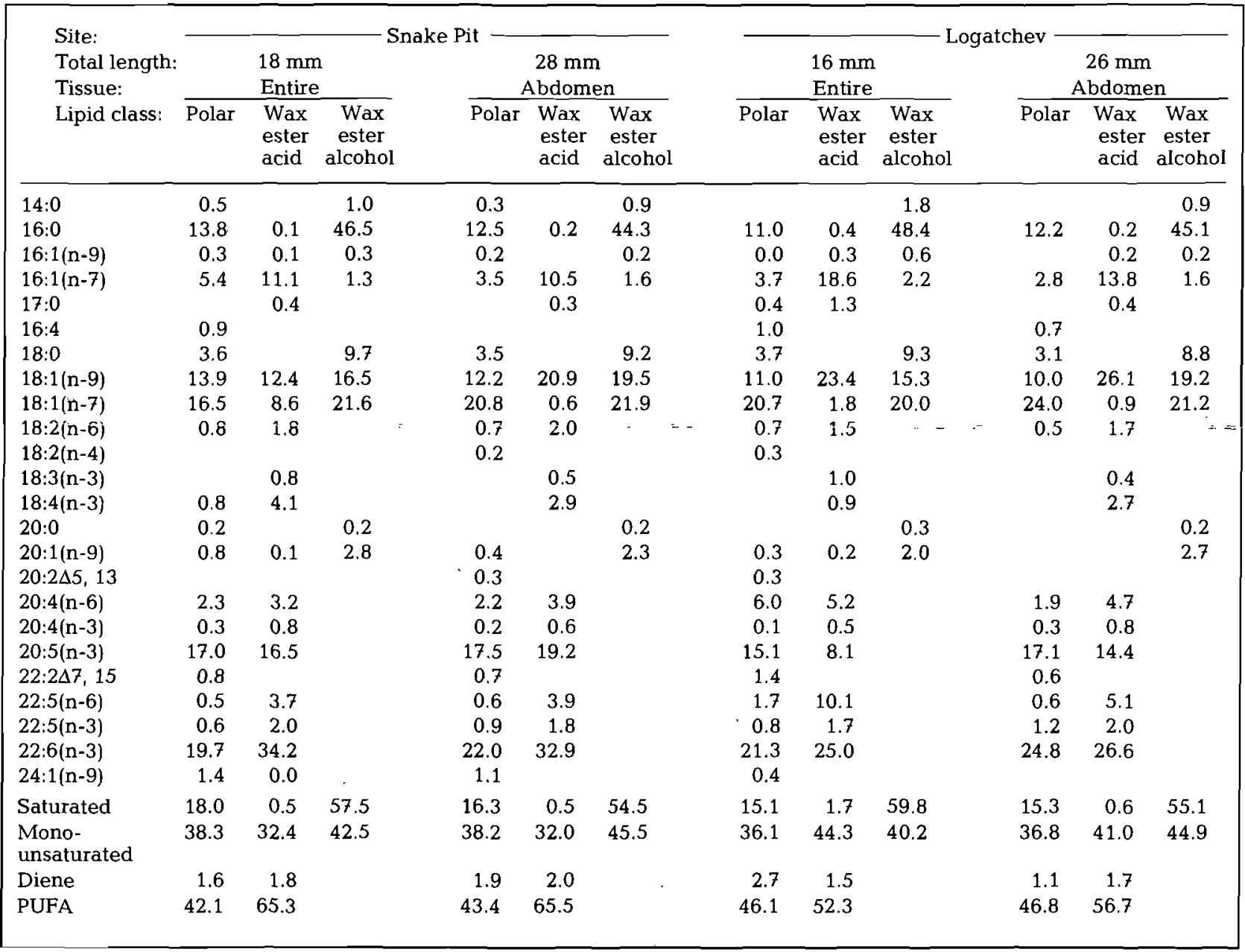

acids in the juvenile shrimp were 20:5(n-3), 18:1(n-7), 18:1(n-9), 16:1(n-7) and 16:0. The fatty alcohol components of the wax esters comprised almost exclusively 16:0, 18:1(n-7) and 18:1(n-9) fatty alcohols (Table 3). The fatty acid composition of the small adult $R$. exoculata was broadly similar to that of the juvenile shrimp, although 22:6(n-3) comprised a higher proportion of fatty acids and was a very substantial component of the wax ester and triacylglycerol lipids (50.2 and $52.1 \%$ respectively, Table 4 ).

Adult Rimicaris exoculata contained minor amounts of wax ester, and thus most fatty acids were contained within the polar and triacylglycerol lipid classes. There were some fundamental differences in the fatty acid profiles of adult shrimp compared with the juvenile and small adult shrimp, with lower proportions of the (n-3) PUFAs 20:5(n-3) and 22:6(n-3). This was particularly evident in triacylglycerols where in some cases only very low levels of (n-3) PUFA were found (Table 5). The fatty acid profiles of adult $R$. exoculata also differed in that they contained substantial amounts of (n-4) and $(\mathrm{n}-7)$ fatty acids and also the nonmethylene interrupted dienes $20: 2 \Delta 5,13$ and 22:2 $\Delta 7,15$ (Table 5).

\section{$\delta^{13} \mathrm{C}$ values}

Stable carbon isotope values of individual fatty acids were highly variable with values ranging from $-6.6 \%$ for $18: 2(\mathrm{n}-4)$ for the $57 \mathrm{~mm}$ adult specimen taken from Broken Spur to $-29.1 \%$ for $16: 1(\mathrm{n}-7)$ in the $53 \mathrm{~mm}$ adult from the Rainbow site. Although $\delta^{13} \mathrm{C}$ values for fatty acids within single shrimp were less variable, there were still substantial differences of up to $11.5 \%$ (53 mm adult from Rainbow, Table 6). $\delta^{13} \mathrm{C}$ values of 20:5(n-3) and 22:6(n-3) in all categories of shrimp varied from -17.1 to $-27.1 \%$. $\delta^{13} \mathrm{C}$ values of the fatty alcohols were less variable with overall maximum and minimum values of -21.0 and $-26.7 \%$ respectively (Tables $7 \&$ ) 
Table 4. Rimicaris exoculata. Fatty acid and fatty alcohol composition (\%) of small adults containing both wax ester and triacylglycerol neutral lipid reserves

\begin{tabular}{|c|c|c|c|c|c|c|c|c|}
\hline \multirow{2}{*}{$\begin{array}{l}\text { Site: } \\
\text { Total length: } \\
\text { Tissue: } \\
\text { Lipid class: }\end{array}$} & \multicolumn{4}{|c|}{$\begin{array}{c}26 \mathrm{~mm} \\
\text { Abdomen }\end{array}$} & \multicolumn{4}{|c|}{$\begin{array}{c}25 \mathrm{~mm} \\
\text { Abdomen }\end{array}$} \\
\hline & $\overline{\text { Polar }}$ & $\begin{array}{c}\text { Triacyl- } \\
\text { glycerol }\end{array}$ & $\begin{array}{c}\text { Wax ester } \\
\text { acid }\end{array}$ & $\begin{array}{l}\text { Wax ester } \\
\text { alcohol }\end{array}$ & Polar & $\begin{array}{l}\text { Triacyl- } \\
\text { glycerol }\end{array}$ & $\begin{array}{c}\text { Wax ester } \\
\text { acid }\end{array}$ & $\begin{array}{c}\text { Wax ester } \\
\text { alcohol }\end{array}$ \\
\hline $14: 0$ & & 0.3 & & 0.5 & & 2.1 & & 2.8 \\
\hline $14: 1$ & & & & & & 0.4 & & \\
\hline $15: 0$ & & 0.2 & & & & & & \\
\hline $16: 0$ & 10.1 & 6.4 & 0.8 & 42.3 & 11.4 & 13.8 & 0.5 & 46.7 \\
\hline $16: 1(n-9)$ & & 1.1 & 0.3 & 0.2 & & 1.2 & 0.6 & 0.4 \\
\hline $16: 1(n-7)$ & 7.2 & 4.7 & 2.1 & 1.1 & 4.6 & 13.7 & 20.4 & 1.8 \\
\hline $16: 1(n-5)$ & & & 1.1 & & & 1.4 & & \\
\hline $16: 2(n-4)$ & 1.4 & 1.0 & & & & 7.0 & & \\
\hline $17: 0$ & & & 1.1 & & & & 0.7 & \\
\hline $16: 4$ & & & & & 1.0 & & & \\
\hline $18: 0$ & & 1.6 & 0.7 & 14.5 & 4.5 & 2.7 & & 9.9 \\
\hline $18: 1(n-9)$ & 12.1 & 4.6 & 4.3 & 16.0 & 13.5 & 13.9 & 25.5 & 16.7 \\
\hline $18: 1(n-7)$ & 19.6 & 4.1 & 0.4 & 22.7 & 22.5 & 10.2 & 1.9 & 18.5 \\
\hline $18: 2(n-6)$ & 0.9 & 0.4 & 0.3 & & 1.0 & 0.7 & 2.1 & \\
\hline $18: 2(n-4)$ & 6.9 & 4.4 & & & 1.5 & 10.9 & & \\
\hline $18: 3(n-7)$ & 1.8 & & & & & 0.7 & & \\
\hline $18: 3(n-3)$ & & & & & & & 1.4 & \\
\hline $18: 4(n-3)$ & & & & & & & 0.7 & \\
\hline $20: 0$ & & & & 0.5 & & & & 0.4 \\
\hline $20: 1(n-9)$ & 0.3 & & & 2.2 & & & & 2.6 \\
\hline $20: 2 \Delta 5,13$ & 1.1 & 0.4 & & & & & & \\
\hline $20: 4(n-6)$ & 8.9 & 4.8 & 5.9 & & 2.1 & 1.4 & 4.8 & \\
\hline $20: 4(n-3)$ & & & 0.2 & & & 0.2 & 0.3 & \\
\hline $20: 5(n-3)$ & 9.7 & 4.5 & 11.7 & & 14.6 & 4.0 & 8.3 & \\
\hline $22: 1$ & & & & & & & & \\
\hline $22: 2 \Delta 7,15$ & 1.1 & & & & 1.1 & 0.4 & & \\
\hline $22: 5(n-6)$ & 1.8 & 6.9 & 17.4 & & & 0.9 & 7.0 & \\
\hline $22: 5(n-3)$ & 0.7 & 2.5 & 3.6 & & & 0.6 & 1.5 & \\
\hline $22: 6(n-3)$ & 16.4 & 52.1 & 50.2 & & 21.8 & 13.7 & 24.3 & \\
\hline $24: 1(n-9)$ & & & & & 0.6 & & & \\
\hline Saturated & 10.1 & 8.5 & 2.6 & 57.8 & 15.9 & 18.6 & 1.2 & 59.8 \\
\hline Monounsaturated & 39.2 & 14.5 & 8.2 & 42.2 & 41.2 & 40.8 & 48.4 & 40.2 \\
\hline Diene & 11.4 & 6.2 & 0.3 & & 3.6 & 19.0 & 2.1 & \\
\hline PUFA & 39.3 & 70.8 & 89.0 & & 39.5 & 21.5 & 48.3 & \\
\hline
\end{tabular}

\section{DISCUSSION}

Deep sea hydrothermal vent environments support abundant, albeit highly localized, animal communities (Childress \& Fisher 1992, Van Dover 1995). The superheated fluids discharged at vent sites are rich in geothermally reduced compounds, primarily hydrogen sulfide and methane, which are oxidized by chemoautotrophic bacteria to provide an energy source to drive the fixation of $\mathrm{CO}_{2}$ and other $\mathrm{C}_{1}$ compounds into organic molecules. The vent animal community then uses the organic metabolites produced by these bacteria as food. Since their discovery, it has generally been accepted that deep sea hydrothermal vent ecosystems are not reliant on processes occurring in the surface layers of the ocean and that the contribution of organic molecules fixed by photosynthetic processes is negligible. However, recent biomarker and stable isotope evidence suggests that some animals associated with the East Pacific (Rieley et al. 1995) and MAR vent sites (Pond et al. 1997a,b,c) can contain high levels of compounds which originate in photosynthetic microplankton.

The life-history strategy of MAR vent shrimp is now reasonably well understood (Dixon et al. 1998, Herring 1998). It is clear that these animals spend a substantial period of their early life stages as planktotrophic organisms, feeding on photosynthetic material and accumulating substantial reserves of neutral lipid before returning to a suitable vent site, prior to maturation (Pond et al. 1997a, Dixon et al. 1998). In the present study, analyses of bacterial/detrital material collected from the vent sites and at the same locations as the 
Table 5. Rimicaris exoculata. Fatty acid composition (\%) of adults which contain only triacylglycerol neutral lipid reserves

\begin{tabular}{|c|c|c|c|c|c|c|c|c|c|c|c|c|}
\hline \multirow{2}{*}{$\begin{array}{l}\text { Site: } \\
\text { Total length: } \\
\text { Tissue: } \\
\text { Lipid class: }\end{array}$} & \multicolumn{2}{|c|}{$\overbrace{\begin{array}{c}53 \mathrm{~mm} \\
\text { Maxilliped }\end{array}}^{\mathrm{Ra}}$} & \multicolumn{2}{|c|}{$\begin{array}{c}20.7 \mathrm{~mm} \\
\text { Abdomen }\end{array}$} & \multicolumn{2}{|c|}{$\begin{array}{c}\text { Broken Spur } \\
57 \mathrm{~mm} \\
\text { Abdomen }\end{array}$} & \multicolumn{2}{|c|}{$\begin{array}{c}\text { TAG } \\
26 \mathrm{~mm} \\
\text { Maxilliped }\end{array}$} & \multicolumn{2}{|c|}{$\begin{array}{l}\text { Snake.Pit } \\
57 \mathrm{~mm} \\
\text { Abdomen }\end{array}$} & \multicolumn{2}{|c|}{$\begin{array}{c}\text { Logatchev } \\
29 \mathrm{~mm} \\
\text { Abdomen }\end{array}$} \\
\hline & $\overline{\text { Polar }}$ & $\begin{array}{l}\text { Triacyl- } \\
\text { glycerol }\end{array}$ & Polar & $\begin{array}{l}\text { Triacyl- } \\
\text { glycerol }\end{array}$ & Polar & $\begin{array}{l}\text { Triacyl- } \\
\text { glycerol }\end{array}$ & $\overline{\text { Polar }}$ & $\begin{array}{l}\text { Triacyl- } \\
\text { glycerol }\end{array}$ & Polar & $\begin{array}{l}\text { Triaçl- } \\
\text { glycerol }\end{array}$ & Polar & $\begin{array}{l}\text { riacyl- } \\
\text { lycerol }\end{array}$ \\
\hline $14: 0$ & & 1.0 & & 0.8 & & 1.6 & & 4.9 & 0.2 & 0.8 & 0.2 & 0.7 \\
\hline $14: 1$ & & 0.4 & & 0.3 & & 0.7 & & 5.3 & & & & \\
\hline $15: 0$ & & 0.3 & & 0.3 & & & & & & & & \\
\hline $16: 0$ & 12.4 & 14.1 & 9.9 & 17.4 & 9.7 & 11.2 & 8.1 & 8.9 & 9.8 & 11.5 & 9.5 & 12.0 \\
\hline $16: 1(n-9)$ & & 1.0 & & 0.7 & & 0.2 & & & 0.1 & 1.5 & & 0.6 \\
\hline $16: 1(n-7)$ & 15.1 & 21.5 & 15.6 & 28.9 & 15.2 & 24.3 & 18.4 & 21.2 & 6.1 & 10.2 & 9.9 & 16.3 \\
\hline $16: 1(n-5)$ & & & & & & & & 2.8 & & & & 1.2 \\
\hline $16: 2(n-4)$ & 0.6 & 2.6 & 0.7 & 3.5 & 1.5 & 5.0 & 8.2 & 15.6 & 0.5 & 2.5 & 1.7 & 6.6 \\
\hline $17: 0$ & & & & & & & & & & & & \\
\hline $16: 4$ & 0.9 & & & 0.9 & & & & 1.2 & & 0.9 & & \\
\hline $18: 0$ & 3.0 & 2.0 & & 1.3 & 2.2 & 1.8 & 3.5 & 0.8 & 5.5 & 2.8 & 4.3 & 3.4 \\
\hline $18: 1(n-9)$ & 8.8 & 11.0 & $=11.8$ & 12.8 & 6.4 & 2.9 & 5.4 & 4.3 & 7.4 & 7.8 & 7.7 & 5.4 \\
\hline $18: 1(n-7)$ & 21.3 & 10.7 & 22.6 & 12.8 & 21.5 & 13.5 & 15.6 & 10.0 & 19.7 & 10.0 & 18.2 & 14.2 \\
\hline $18: 2(n-6)$ & 0.2 & 0.4 & 0.5 & 0.2 & 1.5 & 0.1 & 0.5 & 0.1 & 0.4 & 0.5 & 1.2 & 0.2 \\
\hline $18: 2(n-4)$ & 10.4 & 8.6 & 11.5 & 12.2 & 14.3 & 24.8 & 16.7 & 19.7 & 4.5 & 8.2 & 13.1 & 25.2 \\
\hline $18: 3(n-7)$ & 0.8 & 0.4 & 2.4 & & 9.2 & 8.6 & 0.8 & & 0.9 & 1.7 & 5.8 & 7.8 \\
\hline $20: 0$ & & 0.3 & 0.4 & & & & & & & & 0.2 & \\
\hline $20: 1$ & & & 0.6 & 0.5 & 0.7 & & 0.4 & 0.5 & 0.5 & & 0.8 & 0.3 \\
\hline $20: 2 \Delta 5,13$ & 3.3 & 2.4 & 7.9 & 4.9 & 4.9 & 3.3 & 0.3 & 0.4 & 0.5 & & 2.7 & 1.8 \\
\hline $20: 4(n-6)$ & 1.7 & 2.0 & 3.2 & 0.1 & 0.7 & 0.4 & 5.5 & 0.5 & 5.4 & 7.8 & 1.5 & 0.4 \\
\hline $20: 4(n-3)$ & & 0.2 & & & & & & & 0.2 & 0.5 & & \\
\hline $20: 5(n-3)$ & 7.9 & 3.9 & 5.8 & 0.5 & 4.0 & 0.5 & 6.4 & 0.8 & 14.1 & 8.8 & 8.4 & 1.3 \\
\hline $22: 1$ & & & & & & & 0.2 . & & & & & \\
\hline $22: 2 \Delta 7,15$ & 1.0 & 0.6 & 1.7 & 0.9 & 1.4 & 0.4 & 0.9 & 0.7 & 0.9 & . & 0.9 & 0.3 \\
\hline $22: 5(n-6)$ & 0.5 & 1.4 & 0.5 & 0.2 & 0.6 & & 0.8 & 0.6 & 1.7 & 13.1 & 0.7 & 0.2 \\
\hline $22: 5(n-3)$ & 0.3 & 0.6 & 0.4 & & 0.4 & & 0.3 & 0.2 & 0.8 & 4.0 & 0.7 & \\
\hline $22: 6(n-3)$ & 11.8 & 14.5 & 4.5 & 1.3 & 4.8 & 0.7 & 8.1 & 2.4 & 19.8 & 8.4 & 11.4 & 2.1 \\
\hline $24: 1(n-9)$ & & 0.2 & & 0.3 & & & & 0.1 & & & 0.2 & \\
\hline Saturated & 15.4 & 17.7 & 10.3 & 19.8 & 11.9 & 14.6 & 11.6 & 14.6 & 15.5 & 15.1 & 14.2 & 16.1 \\
\hline Monounsaturated & 45.2 & 44.4 & 50.6 & 56.3 & 43.8 & 40.9 & 40.0 & 44.2 & 33.8 & 29.5 & 36.8 & 38.0 \\
\hline Dienes & 15.5 & 14.6 & 22.3 & 21.7 & 23.6 & 33.6 & 26.6 & 36.5 & 6.8 & 11.2 & 19.6 & 34.1 \\
\hline PUFA & 23.9 & 23.0 & 16.8 & 2.1 & 20.6 & 10.9 & 21.9 & 4.5 & 42.9 & 44.3 & 29.8 & 11.8 \\
\hline
\end{tabular}

Table 6. Rimicanis exoculata. Stable carbon isotope values $\left(\delta^{13} \mathrm{C} \% \mathrm{v}\right.$-PDB) of the fatty acids and fatty alcohols contained in the major lipid classes of adults

\begin{tabular}{|c|c|c|c|c|c|c|c|c|c|c|c|c|}
\hline \multirow{3}{*}{$\begin{array}{l}\text { Site: } \\
\text { Total length: } \\
\text { Tissue: } \\
\text { Lipid class: }\end{array}$} & \multicolumn{4}{|c|}{ - Rainbow } & \multirow{2}{*}{\multicolumn{2}{|c|}{$\begin{array}{l}\text { Broken Spur } \\
57 \mathrm{~mm} \\
\text { Abdomen }\end{array}$}} & \multirow{2}{*}{\multicolumn{2}{|c|}{$\begin{array}{c}\text { TAG } \\
26 \mathrm{~mm} \\
\text { Maxilliped }\end{array}$}} & \multirow{2}{*}{\multicolumn{2}{|c|}{$\begin{array}{l}\text { Snake Pit } \\
57 \mathrm{~mm} \\
\text { Abdomen }\end{array}$}} & \multirow{2}{*}{\multicolumn{2}{|c|}{$\begin{array}{c}\text { Logatchev } \\
29 \mathrm{~mm} \\
\text { Abdomen }\end{array}$}} \\
\hline & \multicolumn{2}{|c|}{$\begin{array}{l}53 \mathrm{~mm} \\
\text { Maxilliped }\end{array}$} & \multicolumn{2}{|c|}{$\begin{array}{c}20.7 \mathrm{~mm} \\
\text { Abdomen }\end{array}$} & & & & & & & & \\
\hline & $\overline{\text { Polar }}$ & $\begin{array}{l}\text { Triacyl- } \\
\text { glycerol }\end{array}$ & Polar & $\begin{array}{l}\text { Triacyl- } \\
\text { glycerol }\end{array}$ & Polar & $\begin{array}{l}\text { Triacyl- } \\
\text { glycerol }\end{array}$ & $\overline{\text { Polar }}$ & $\begin{array}{l}\text { Triacyl- } \\
\text { glycerol }\end{array}$ & $\overline{\text { Polar }}$ & $\begin{array}{l}\text { Triacyl- } \\
\text { glycerol }\end{array}$ & $\begin{array}{r}\overline{\text { Polar }} \\
g\end{array}$ & $\begin{array}{l}\text { Triacyl- } \\
\text { ylycerol }\end{array}$ \\
\hline $16: 0$ & -20.2 & -18.8 & -21.2 & -17.8 & -12.1 & -14.0 & -17.2 & -16.0 & -16.2 & -22.8 & -15.7 & -16.9 \\
\hline $16: 1(n-7)$ & -29.1 & -14.5 & -11.2 & -12.4 & -8.4 & -8.8 & -12.4 & -13.5 & -17.6 & -22.3 & -12.8 & -13.1 \\
\hline $16: 2(n-4)$ & & -16.8 & -10.4 & -12.5 & -7.9 & -8.8 & -11.2 & -13.8 & -17.5 & -21.4 & -14.1 & -14.7 \\
\hline $18: 0$ & & -22.3 & & -20.5 & -11.2 & -13.2 & & & & & & -18.5 \\
\hline $18: 1(n-9)$ & -19.1 & -21.3 & -18.2 & -18.4 & -12.1 & -11.1 & -17.4 & -19.1 & -18.9 & -24.6 & -18.7 & -20.3 \\
\hline $18: 1(n-7)$ & -17.7 & -13.7 & -17.4 & -11.7 & -10.2 & -9.2 & -11.5 & -15.7 & -18.5 & -21.3 & -14.3 & -14.6 \\
\hline $18: 2(n-4)$ & & -14.5 & -11.1 & -11.8 & -11.1 & -6.6 & -12.1 & -14.8 & -17.7 & & -11.7 & -13.7 \\
\hline $20: 2 \Delta 5,13$ & & -16.8 & -16.7 & -17.9 & -10.3 & -11.0 & & & & & & \\
\hline $20: 4(n-6)$ & & & & & & & -19.5 & & -22.1 & -21.2 & & \\
\hline $20: 5(n-3)$ & -17.6 & -17.8 & -18.1 & & -18.4 & & -19.2 & & -18.3 & -20.4 & -20.3 & \\
\hline $22: 5(n-6)$ & & & & & & & & & & -23.3 & & \\
\hline $22: 6(n-3)$ & -22.4 & -22.3 & -21.7 & & -17.1 & & -16.8 & & -24.2 & -19.5 & -19.6 & \\
\hline
\end{tabular}


Table 7. Rimicaris exoculata. Stable carbon isotope values $\left(\delta^{13} \mathrm{C} \% \mathrm{v}-\mathrm{PDB}\right)$ of fatty acids and fatty alcohols contained in the major lipid classes of 'orange' (16 to $18 \mathrm{~mm}$ ) and small adults (26 to $28 \mathrm{~mm}$ ) ["indicates where 18:1(n-9) and 18:1(n-7) are unresolved]

\begin{tabular}{|c|c|c|c|c|c|c|c|c|c|c|c|c|}
\hline \multirow{3}{*}{$\begin{array}{l}\text { Site: } \\
\text { Total length: } \\
\text { Tissue: } \\
\text { Lipid class: }\end{array}$} & \multicolumn{6}{|c|}{ Snake Pit } & \multirow{2}{*}{\multicolumn{3}{|c|}{$\begin{array}{l}16 \mathrm{~mm} \\
\text { Entire }\end{array}$}} & \multirow{2}{*}{\multicolumn{3}{|c|}{$\begin{array}{c}26 \mathrm{~mm} \\
\text { Abdomen }\end{array}$}} \\
\hline & \multicolumn{3}{|c|}{$\begin{array}{l}18 \mathrm{~mm} \\
\text { Entire }\end{array}$} & \multicolumn{3}{|c|}{$\begin{array}{c}28 \mathrm{~mm} \\
\text { Abdomen }\end{array}$} & & & & & & \\
\hline & Polar & $\begin{array}{l}\text { Wax } \\
\text { ester } \\
\text { acid }\end{array}$ & $\begin{array}{c}\text { Wax } \\
\text { ester } \\
\text { alcohol }\end{array}$ & Polar & $\begin{array}{l}\text { Wax } \\
\text { ester } \\
\text { acid }\end{array}$ & $\begin{array}{c}\text { Wax } \\
\text { ester } \\
\text { alcohol }\end{array}$ & $\overline{\text { Polar }}$ & $\begin{array}{l}\text { Wax } \\
\text { ester } \\
\text { acid }\end{array}$ & $\begin{array}{c}\text { Wax } \\
\text { ester } \\
\text { alcohol }\end{array}$ & Polar & $\begin{array}{l}\text { Wax } \\
\text { ester } \\
\text { acid }\end{array}$ & $\begin{array}{c}\text { Wax } \\
\text { ester } \\
\text { alcohol }\end{array}$ \\
\hline $16: 0$ & -20.2 & & -22.0 & -18.9 & & -21.9 & -20.4 & & -24.8 & -20.3 & & -21.0 \\
\hline $16: 1(n-7)$ & -29.0 & -23.9 & & -19.7 & -20.9 & & & -22.3 & & -21.2 & -19.4 & \\
\hline 18:0 & & & -23.2 & & & -21.5 & & & -23.2 & & & -25.0 \\
\hline $18: 1(n-9)$ & -19.1 & -18.8 & -21.7 & $-18.4^{*}$ & -20.2 & -26.0 & $-22.6^{*}$ & -20.3 & -23.8 & $-20.4^{*}$ & -21.1 & -25.4 \\
\hline $18: 1(n-7)$ & -17.7 & -15.2 & -21.8 & & & -24.0 & & -20.0 & -23.3 & & & -21.9 \\
\hline $20: 4(n-6)$ & & & & & -25.9 & & -18.3 & & & & & \\
\hline $20: 5(n-3)$ & -21.4 & -21.2 & & -22.3 & -23.8 & & -21.1 & -19.8 & & -20.5 & -20.0 & \\
\hline $22: 6(n-3)$ & -22.4 & -20.4 & & -21.9 & -27.1 & & -21.7 & -19.9 & & -22.1 & -19.3 & \\
\hline
\end{tabular}

Table 8. Rimicaris exoculata. Stable carbon isotope values $\left(\delta^{13} \mathrm{C} \% \mathrm{v}-\mathrm{PDB}\right)$ of the major fatty acids and fatty alcohols in small adults

\begin{tabular}{|c|c|c|c|c|c|c|c|c|}
\hline \multirow{3}{*}{$\begin{array}{l}\text { Site: } \\
\text { Total length: } \\
\text { Tissue: } \\
\text { Lipid class: }\end{array}$} & \multirow{2}{*}{\multicolumn{4}{|c|}{$\begin{array}{c}26 \mathrm{~mm} \\
\text { Abdomen }\end{array}$}} & & & & \\
\hline & & & & & \multicolumn{4}{|c|}{$\begin{array}{c}25 \mathrm{~mm} \\
\text { Abdomen }\end{array}$} \\
\hline & $\overline{\text { Polar }}$ & $\begin{array}{l}\text { Triacyl- } \\
\text { glycerol }\end{array}$ & $\begin{array}{l}\text { Wax ester } \\
\text { acid }\end{array}$ & $\begin{array}{c}\text { Wax ester } \\
\text { alcohol }\end{array}$ & Polar & $\begin{array}{l}\text { Triacyl- } \\
\text { glycerol }\end{array}$ & $\begin{array}{c}\text { Wax ester } \\
\text { acid }\end{array}$ & $\begin{array}{c}\text { Wax ester } \\
\text { alcohol }\end{array}$ \\
\hline $16: 0$ & -20.2 & -22.2 & -27.3 & -23.0 & -23.6 & -24.1 & -29.0 & -25.4 \\
\hline $16: 1(n-7)$ & -17.2 & -18.7 & -25.8 & & -20.8 & -22.3 & -26.3 & \\
\hline $18: 0$ & -24.3 & -26.2 & -28.0 & -26.7 & & & & -25.6 \\
\hline $18: 1(n-9)$ & -19.2 & -22.0 & -25.2 & -24.0 & -20.6 & -21.5 & -23.2 & -25.6 \\
\hline $18: 1(n-7)$ & -21.5 & -20.9 & -23.8 & -24.8 & -17.0 & -17.6 & -20.8 & -26.7 \\
\hline $18: 2(n-4)$ & -18.2 & -18.4 & & & & -17.2 & & \\
\hline $20: 4(n-6)$ & -20.5 & -23.7 & -21.1 & & -24.8 & -25.6 & -24.8 & \\
\hline $20: 5(n-3)$ & -22.6 & -24.7 & -25.5 & & -22.6 & -24.7 & -23.9 & \\
\hline $22: 5(n-6)$ & & -23.7 & -23.3 & & & & -22.7 & \\
\hline $22: 6(n-3)$ & -17.9 & -17.6 & -22.6 & & -20.7 & -23.1 & -22.6 & \\
\hline
\end{tabular}

samples of shrimp showed only very low amounts of PUFA which supports the contention that these same compounds in the shrimp are derived from photosynthetic microplankton.

The detailed analyses of the neutral lipid reserves contained in the juvenile and small adult Rimicaris exoculata have established that they contain extraordinarily high levels of PUFA (up to $89 \%$ of total fatty acids, Tables 3 to 5). In marine animals which are known to accumulate triacylglycerol or wax esters as reserve lipids, the fatty acids comprising these compounds are predominantly saturated (no double bonds) or monounsaturated fatty acids (a single double bond) and PUFA are often only minor components (Sargent \& Henderson 1995). Thus, the high levels of PUFA contained in the neutral lipids of $R$. exoculata are considerably higher than those previously reported for marine Crustacea (Falk-Petersen et al. 1987, Norrbin et al. 1990, Sargent and Henderson 1995, Kattner et al.
1996). GC-IRMS analyses indicate that the $\delta^{13} \mathrm{C}$ values of these PUFA contained in neutral lipid are from -17.6 to $-27.1 \%$, which is within the range consistent with a photosynthetic origin of these compounds and supports previous studies (Pond et al. 1997a,b,c, in press). We believe that these PUFA are selectively accumulated by the shrimp during their early planktonic life stage before subsequent mobilization into phospholipids as and when required to sustain growth and maturation of the shrimp into adulthood. This selective incorporation of dietary PUFA into wax ester reserves during the planktotrophic life stage must ultimately be genetically regulated, which indicates that $R$. exoculata has evolved a highly specialized lipid metabolism adapted to its life cycle.

There is a clear progression from a lipid profile in the juvenile shrimp which is dominated by wax esters and PUFA, to the small adult which contains both wax ester and triacylglycerol and finally to the adult shrimp with 
triacylglycerol and the presence of bacterial signature fatty acids [16:2(n-4); 18:2(n-4); 20:2 55,$13 ; 22: 2 \Delta 7,15]$. This progression in the lipid composition is entirely consistent with the 'orange' shrimp being a juvenile stage of Rimicaris exoculata. The transition between a neutral lipid profile which is dominated by wax esters during the planktotrophic stage and triacylglycerols after maturation (Fig. 1, and Copley et al. 1998) is unusual as most lipid-rich marine animals tend to store a single neutral lipid class throughout their life cycle. Wax esters are considered to be long term metabolic energy reserves and are characteristic of animals which experience marked seasonality in the availability of their food (Lee \& Hirota 1973). Wax ester reserves have also been implicated in providing buoyancy which would be beneficial for planktonic animals as it could plausibly, reduce the metabolic costs associated with swimming (Sargent 1976). Although the specific gravity of lipid is lower than unity, there are considerable differences between the various lipid classes. The specific gravity of wax ester is approximately 0.86 which would provide an upthrust of $0.193 \mathrm{~g} \mathrm{~g}^{-1}$ lipid (Sargent 1976). Triacylglycerols, with a specific gravity of 0.93 , would provide an upthrust force of only $0.103 \mathrm{~g}$ $\mathrm{g}^{-1}$ lipid and may therefore be the preferred neutral reserve lipid for a benthic animal which swims and feeds immediately adjacent to buoyant hydrothermal plumes. However, it is clear that whatever the reason for the transition from wax ester to triacylglycerol storage lipids during maturation, the mobilization of PUFA from wax ester into triacylglycerols again points to a highly regulated and evolved lipid metabolism.

It is not within the scope of this study to compare differences in the lipid and stable carbon isotope values of shrimp between vent sites, but it is obvious that the isotope signatures of the fatty acids are highly variable; as has previously been observed for biological material collected from hydrothermal vent fields (Fisher et al. 1994, Van Dover \& Fry 1994, Pond et al. 1997c). The $\dot{\delta}^{13} \mathrm{C}$ values of the fatty alcohols were generally less variable than those of the fatty acids. The ranges of $\delta^{13} \mathrm{C}$ values in photosynthetic systems are usually much less variable than those in chemoautotrophic organisms (Goericke et al. 1994), which suggests that the fatty alcohols are derived from a photosynthetic carbon source.

Given the widespread distribution of Rimicaris exoculata along the MAR, coupled with their potentially high population densities (Gebruk et al. 1997), these animals must be responsible for a substantial and direct transport of PUFA into the hydrothermal vent ecosystem. The wider ecological implications of this 'import' of PUFA into the vent ecosystem are unknown. Organisms such as MAR vent mussels appear not to require high levels of PUFA, as their tissues contain only very low amounts of these compounds (Pond et al. 1998). However, any fish populations associated with the MAR vent sites would undoubtedly require a dietary input of PUFA as these compounds are essential for vertebrate nutrition (Sargent et al. 1995).

In summary, it is clear from the above findings that the life-history strategy and nutritional biochemistry of Rimicaris exoculata are highly specialized and have evolved specifically to enable this shrimp species to obtain and store substantial amounts of PUFA produced by phytoplankton. Thus, although these shrimp are similar to the vast majority of other animals which inhabit the marine environment, in that they are to a degree dependent on organic compounds produced in the surface layers of the ocean, they are also able to exploit successfully the abundant chemosynthetic food resources available at the deep water MAR vent sites.

Acknowledgements. The collection of samples, including the cost of submersible time, was financed by the UK Natural Environment Research Council (NERC) as part of the British MidOcean Ridge Initiative (BRIDGE), Grant No. GST/02/1478 to A.J.S. We thank Dr D. Fornari of the Woods Hole Oceanographic Institution for his help in arranging participation in the 'Atlantis' cruise and Professors R. C. Vrijenhoek and R. A. Lutz of Rutgers University for their willingness to share the second leg of the cruise. Dr T. Shank of Rutgers University co-operated in the onboard identification of shrimp, and Dr A. Vereshchaka of the Shirshov Institute of Oceanography kindly shared his latest data on shrimp species. The Isotope Geosciences Unit at the Scottish Universities Research and Reactor Centre, East Kilbride, is supported by a consortium of Scottish Universities and the NERC.

\section{LITERATURE CITED}

Abrajano TA Jr, Murphy DE, Fang J, Comet $\mathrm{P}$, Brooks JM (1994) ${ }^{13} \mathrm{C}^{12} \mathrm{C}$ ratios of individual fatty acids of marine mytilids with and without bacterial symbionts. Org Geochem 21:611-617

Childress JJ, Fisher CR (1992) The biology of the hydrothermal vent animals: physiology, biochemistry and autotrophic symbioses. Oceanogr Mar Biol Annu Rev 30:337-441

Copley C, Tyler P, Varney M (1998) Lipid profiles of hydrothermal vent shrimps. Cah Biol Mar 34:229-231

Dixon DR, Dixon LRJ, Pond DW (1998) Recent advances in our understanding of the life history of bresiliid vent shrimps on the MAR. Cah Biol Mar 39:383-386

Eakin PA, Fallick AE, Gerc J (1992) Some instrumental effects in the determination of stable isotope ratios by gas chromatography isotope ratio mass spectrometry. Chem Geol 101:71-79

Falk-Petersen S, Sargent JR, Tande KS (1987) Lipid composition of zooplankton in relation to the sub-Arctic food web. Polars Biol 8:115-120

Farquhar JW (1962) Identification and gas-liquid chromatographic behaviour of plasmalogen aldehydes and their acetal, alcohol and acetylated alcohol derivatives. J Lipid Res 3:21-30

Fisher CR, Childress JJ, Macko SA, Brooks JM (1994) Nutritional interactions in Galapagos Rift hydrothermal vent 
communities: inferences from stable carbon isotope analyses. Mar Ecol Prog Ser 103:45-55

Folch J, Lees N, Sloan-Stanley GH (1957) A simple method for the isolation and purification of total lipid. J Biol Chem 226:497-509

Gebruk AV, Galkin SV, Vereshchaka AL, Moskalev LL, Southward AJ (1997) Ecology and biogeography of the hydrothermal vent fauna of the Mid-Atlantic Ridge. Adv Mar Biol 32:93-144

Goericke R, Montoya JP, Fry B (1994) Physiology of isotopic fractionation in algae and cyanobacteria. In: Lajtha $\mathrm{K}$, Michener RH (eds) Stable isotopes in marine ecology and environmental science. Blackwell Scientific Publications, Oxford, p 187-221

Herring PJ (1998) North Atlantic midwater distribution of the juvenile stages of hydrothermal vent shrimps (Decapoda: Bresilidae). Cah Biol Mar 39:387-390

Jónasdóttir JH (1994) Effects of food quality on the reproductive success of Acartia tonsa and Acartia hudsonica: laboratory observations. Mar Biol 121:67-81

Kanazawa A, Teshima S, Ono K (1979) Relationship between essential fatty acid requirements of aquatic animals and the capacity for bioconversion of linolenic acid to highly unsaturated fatty acids. Comp Biochem Physiol B 63: 295-298

Kattnèr G, Hagen W, Falk-Petersen S, Sargent JR, Henderson RJ (1996) Antarctic krill Thysanoessa macrura fills a major gap in marine lipogenic pathways. Mar Ecol Prog Ser 134: 295-298

Lee RF, Hirota J (1973) Wax esters in tropical zooplankton and nekton and the geographical distribution of wax esters in marine copepods. Limnol Oceanogr 18:227-239

Norrbin MF, Olsen RE, Tande KS (1990) Seasonal variation in lipid class and fatty acid composition of two small copepods in Balsfjorden, northern Norway. Mar Biol 105: 205-211

Olsen RE, Henderson RJ (1989) The rapid analysis of neutral and polar marine lipids using double development HPTLC and scanning densitometry. J Exp Mar Biol Ecol 129: 189-197

Polz MF, Robinson JJ, Cavanaugh C, Van Dover CL (1998) Trophic ecology of massive shrimp aggregations at a MidAtlantic Ridge hydrothermal vent site. Limnol Oceanogr 43:1631-1638

Pond DW, Harris RP, Head R, Harbour D (1996) Environmental and nutritional factors determining the seasonal variability in the fecundity and egg viability of Calanus helgolandicus in coastal waters off Plymouth, UK. Mar Ecol Prog Ser 143:45-63

Pond DW, Dixon DR, Sargent JR (1997a) Wax-ester reserves facilitate dispersal of hydrothermal vent shrimps. Mar Ecol Prog Ser 146:289-290

Pond DW, Bell MV, Dixon DR, Sargent JR (1997b) Occurrence of $16: 2(n-4)$ and $18: 2(n-4)$ fatty acids in the lipids of the hydrothermal vent shrimp Rimicaris exoculata: nutritional and trophic implications. Mar Ecol Prog Ser 156:167-174

Editorial responsibility: Otto Kinne (Editor),

Oldendorf/Luhe, Germany
Pond DW, Segonzac M, Bell MV, Dixon DR, Fallick AE, Sargent JR (1997c) Lipid and lipid carbon stable isotope composition of the hydrothermal vent shrimp Mirocaris fortunata: evidence for nutritional dependence on photosynthetically-fixed carbon. Mar Ecol Prog Ser 157: 221-231

Pond DW, Bell MV, Dixon DR, Fallick AE, Segonzac M, Sargent JR (1998) Stable carbon isotope composition of fatty acids in hydrothermal vent mussels containing methanotrophic and thiotrophic bacterial endosymbionts. Appl Environ Microbiol 64:370-375

Pond DW, Sargent JR, Fallick AE, Copley C, Bell MV, Dixon DR (2000) $\delta^{13} \mathrm{C}$ values of lipids from phototrophic zone microplankton and bathypelagic shrimps at the Azores sector of the Mid-Atlantic Ridge. Deep-Sea Res Part I 47:121-136

Rieley G, Van Dover CL, Hedrick DB, White DC, Eglinton G (1995) Lipid characteristics of hydrothermal vent organisms from $9^{\circ} \mathrm{N}$ East Pacific Rise. ln: Parson LM, Walker CL, Dixon DR (eds) Hydrothermal vents and processes. The Geological Society, London, p 329-334

Russell NJ, Nichols DS (1999) Polyunsaturated fatty acids in marine bacteria - a dogma rewritten. Microbiology 145: $767-779$

Sargent JR (1976) The structure, metabolism and function of lipids in marine organisms. In: Malins DC, Sargent JR (eds) Biochemical and biophysical perspectives in marine biology. Academic Press, London، p 33-65

Sargent JR, Henderson RJ (1995) Marine (n-3) polyunsaturated fatty acids. In: Hamilton $\mathrm{RJ}$ (ed) Developments in oils and fats. Blackie Academic and Professional, Glasgow, p 33-65

Sargent JR, Bell MV, Bell JG, Henderson RG, Tocher DR (1995) Origins and functions of $n-3$ polyunsaturated fatty acids in marine organisms. In: Ceve $G$, Paltauf $F$ (eds) Phospholipids characterisation, metabolism and novel biological applications. American Oil Society Press, Champaign, IL, p 248-259

Shank TM, Lutz RA, Vrijenhoek RC (1998) Molecular systematics of shrimp (Decapoda: Bresiliidae) from deep-sea hydrothermal vents, I: Enigmatic 'small orange' shrimp from the Mid-Atlantic Ridge are juvenile Rimicaris exocu. lata. Mol Mar Biol Biotechnol 7:88-96

Sofer Z (1980) Preparation of carbon dioxide for stable-isotope analysis of petroleum fractions. Anal Chem 52:1389-1391

Van Dover CL (1995) Ecology of Mid-Atlantic Ridge hydrothermal vents. In: Parson LM, Walker CL, Dixon DR (eds) Hydrothermal vents and processes. The Geological Society, London, p 257-294

Van Dover CL, Fry B (1994) Microorganisms as food resources at deep-sea hydrothermal vents. Limnol Oceanogr 39: 51-57

Vereshchaka AL (1996) A new genus and species of caridean shrimp (Crustacea: Decapoda: Alvinocarididae) from the North Atlantic hydrothermal vents. J Mar Biol Assoc UK 76:951-961

Submitted: September 16, 1999; Accepted: December 9, 1999 Proofs received from author(s): May 11, 2000 